\title{
A q-parameter bound for particle spectra based on black hole thermodynamics with Rényi-entropy
}

\author{
Tamás S. Biróa, Viktor G. Czinner ${ }^{\mathrm{a}, \mathrm{b}}$ \\ ${ }^{a}$ HAS Wigner Research Centre for Physics, Institute for Particle and Nuclear Physics, H-1525 Budapest, P.O.Box 49, Hungary \\ ${ }^{b}$ Centro de Matemática, Universidade do Minho, Campus de Gualtar, 4710-057 Braga, Portugal
}

\begin{abstract}
By regarding the Hawking-Bekenstein entropy of Schwarzschild black hole horizons as a non-extensive Tsallis entropy, its formal logarithm, the Rényi entropy, is considered. The resulting temperature - horizon-radius relation has the same form as the one obtained from a 3+1-dimensional black hole in anti-de Sitter space using the original entropy formula. In both cases the temperature has a minimum. A semi-classical estimate of the horizon radius at this minimum leads to 'a Bekenstein bound for the $q$-parameter in the Rényi entropy of micro black holes, $\left(q \geq 1+2 / \pi^{2}\right)$, which is surprisingly close to fitted $q$-parameters of cosmic ray spectra and power-law distribution of quarks coalescing to hadrons in high 'energy accelerator experiments.
\end{abstract}

Keywords: Non-extensive entropy, Black hole thermodynamics, Heavy ion collisions

\section{Introduction}

Non-extensive thermodynamics [1] aims at describing dynamically and statistically entangled systems. It is noteworthy that already the ideal gas, a constant heat capacity system, shows a power-law tailed distribution of the en"ergy in the microcanonical treatment. In fact the Tsallis entropy can be viewed as

$$
S_{\text {Tsallis }}=K\left(S_{\text {Renyi }}\right)=\sum_{i} p_{i} K\left(-\ln p_{i}\right),
$$

with

$$
K(S)=\frac{1}{a}\left(e^{a S}-1\right) .
$$

In general $a$ is a real parameter, for an ideal gas in particular, and $a=1 / C_{0}$ with $C_{0}$ being the constant, positive heat-capacity [2, 3]. $K(S)$ is additive for the finite ideal gas, while $S$ is not. In the $C_{0} \rightarrow \infty$ limit $a=0$ and the Boltzmann-Gibbs formula follows. It is, in general, a better approximation to consider a constant, but finite value for the parameter $a$.

By these definitions the familiar Boltzmann entropy is given as the expectation value of the 'surprise' measure, $\mathfrak{s}=-\ln p_{i}$ :

$$
S_{B}=-\sum p_{i} \ln p_{i}=\langle\mathfrak{s}\rangle,
$$

while the Rényi entropy is the logarithm of the characteristic function:

$$
S_{R}=\frac{1}{a} \ln \sum p_{i}^{1-a}=\frac{1}{a} \ln \left\langle e^{a \mathfrak{s}}\right\rangle .
$$

Email addresses: biro.tamas@wigner.mta.hu (Tamás S. Biró), czinner.viktor@wigner.mta.hu (Viktor G. Czinner)
The latter generates an infinite series based on the central moments (statistical correlations):

$$
S_{R}=\langle\mathfrak{s}\rangle+\frac{a}{2 !} \delta_{2} \mathfrak{s}+\frac{a^{2}}{3 !} \delta_{3} \mathfrak{s}+\ldots
$$

Usually all central moments scale with the volume $V$ of the system, and interpreting $a=1 / C_{0}$ as discussed and cited above, it scales like $1 / V$. This way the Rényi entropy converges to the Boltzmannian formula as fast as $V^{2-n} / n$ !.

The Tsallis entropy, on the other hand, is given as

$$
S_{T}=K\left(S_{R}\right)=\frac{1}{a}\left(\left\langle e^{a \mathfrak{s}}\right\rangle-1\right),
$$

and it generates a series based on the direct statistical moments, with the generic term being $a^{n-1}\left\langle\mathfrak{s}^{n}\right\rangle / n$ !. All terms in this series are of the same order of $V$. So for fast convergence to the classical results the Rényi entropy is preferable, although, it is only the Tsallis formula which is linear in a direct expectation value based on the $p_{i}$ probabilities, offering in this way advantages for transport theory and other linear response treatments. Both formulas are stable close enough to the entropy maximum - there is no difference from this point of view in equilibrium calculations, which we shall pursue in this paper.

By using a non-Boltzmannian formula for the entropy, the experimentally observed power-law tailed particle spectra can be interpreted as reflecting a canonical ensemble in the non-extensive thermodynamics $[4,[5,66,7]$. The theoretical prediction of the power in single-particle spectra, related to the Tsallis parameter, $q=1+a$, is however a very difficult problem; it requires a physical model of the heat-container present at the source of particle emission. Simplified models, such as dual black holes, became popular lately in this respect [8, 9, 10, 11].

August 7, 2018 
In earlier works we have fitted several hadron spectra observed at RHIC 12, 13, 14, 15, 16, 17, 18] by assuming a blast wave picture and quark recombination [19, 20]. These fits agreed with a common Tsallis parameter of $q \approx$ 1.2 for the quark matter quite remarkably. According to the quark coalescence picture the corresponding value of $(q-1)$ for mesons should be the half, and for baryons one third of this fit.

A power-law tailed energy-distribution can be derived as a canonical distribution stemming either from the Tsallis $\left(S_{T}\right)$ or from the Rényi-entropy $\left(S_{R}\right)[21,22,23,24,25$, 26. Both entropy formulas contain a $q=1+a$ parameter, and, from eqs. (4) and (6), are in fact connected as

$$
S_{R}=K^{-1}\left(S_{T}\right)=\frac{1}{a} \ln \left(1+a S_{T}\right) .
$$

In the $a \rightarrow 0$ limit $S_{R}$ coincides with Boltzmann's entropy formula. The canonical energy distribution is derived from maximizing

$$
S_{R}-\beta \sum_{i} p_{i} E_{i}-\alpha \sum_{i} p_{i} .
$$

Differentiation with respect to $p_{i}$ leads to

$$
p_{i}=A\left(\alpha+\beta E_{i}\right)^{-1 / a}
$$

with the proportionality constant

$$
A=e^{S_{R}}\left(\frac{a}{1-a}\right)^{-1 / a} .
$$

Here $\alpha$ and $\beta$ can be expressed by the other parameters according to the normalization condition $\sum_{i} p_{i}=1$ and the average energy.

In this paper we attempt to obtain an estimate for the $a=q-1$ parameter of the Rényi and Tsallis entropy by investigating simple, static Schwarzschild black hole thermodynamics with maximizing the Rényi logarithm of the Bekenstein-Hawking horizon-entropy. As a result we obtain an interesting thermodynamical behavior which is very similar to the one of a black hole in anti-de Sitter (AdS) space [27], with equation of state obtained by maximizing the original Bekenstein-Hawking entropy, proportional to the horizon area [28, 29, 30]. By an explicit comparison we demonstrate that the resulting thermodynamic temperature, $T=d E / d S$, follows an analogous dependence on the horizon radius in both systems. This offers a re-interpretation of the parameter $a$ in terms of the anti-de Sitter curvature parameter, $\lambda$.

For positive $a$ the resulting temperature has a minimum at a certain horizon radius, both smaller and larger black holes appear hotter and decay faster. By estimating this minimum-temperature radius based on the ground state energy of a semi-classical string spanned over the diameter of the horizon, $2 r=4 E$ in Planck units, we consider the Bekenstein limit 31] for quantum sized black holes. Astronomical black holes on the other hand should posses huge heat capacity, so correspondingly a much smaller magnitude for the parameter $a=1 / C_{0}$. Accordingly, one can expect that the minimum-temperature radius is larger than the size of the largest observed black-hole horizon, so the Tsallis-Rényi parameter is largely different for astronomical and for elementary objects.

All over this paper energy, mass and momentum is measured in multiples of the Planck mass, $M_{P}$, while length and time in multiples of the Planck length, $L_{P}$. All equations relating unlike quantities are to be supported by corresponding powers of $M_{P}$ and $L_{P}$. The entropy is measured in units of the Boltzmann constant, $k_{B}$. In a $c=1, k_{B}=1$ system one expresses the Planck constant as $\hbar=L_{P} M_{P}$ and Newton's gravity constant as $G=L_{P} / M_{P}$.

\section{Bekenstein-Hawking and Rényi entropy for black hole horizons}

The Bekenstein-Hawking entropy for simple (spherically symmetric, static) black hole horizons can easily be obtained as follows. In general for a metric given by

$$
d s^{2}=f(r) d t^{2}-\frac{d r^{2}}{f(r)}-r^{2} d \Omega^{2},
$$

with $t$ and $r$ being the time and radial coordinates for the far, static observer and $d \Omega$ the two-dimensional surface angle, the corresponding Unruh temperature 32] at the horizon becomes

$$
T=\frac{1}{4 \pi} f^{\prime}(r) .
$$

Considering now that the internal energy is practically the mass of the black hole, one obtains the entropy according to Clausius' formula as an integral,

$$
S=4 \pi \int \frac{d M}{f^{\prime}(r)} .
$$

This result can be written in a more elegant form by noting that the denominator, $f^{\prime}(r)$ - to be evaluated at the condition $f(r)=0-$ is a Jacobian for a Dirac-delta constraint. Therefore the above BH-entropy equals to

$$
S=4 \pi \iint \delta(f(r, M)) d r d M .
$$

This form reminds to a microcanonical shell in the phase space of the variables $r$ and $M=E$.

In the case of the Schwarzschild black hole solution one has

$$
f(r)=1-\frac{2 M}{r}
$$

the horizon condition $f(r)=0$ is satisfied at $r=2 M$ and the BH-entropy becomes

$$
S_{B H}=\pi r^{2} .
$$

Since the same result emerges for any $f(r, M)=1-$ $2 M / r-h(r)$, linear in $M$, the entropy of such simple 
black hole horizons is always proportional to their area. So the above "area law" is also valid for example for the Schwarzschild - anti-de Sitter metric, too.

As it is known, the Bekenstein-Hawking result leads to an equation of state, $S(E)$ which describes an object with negative heat capacity:

$$
\begin{aligned}
S_{B H} & =4 \pi E^{2}, \\
\frac{1}{T_{B H}} & =S_{B H}^{\prime}(E)=8 \pi E, \\
C_{B H} & =\frac{-S_{B H}^{\prime 2}(E)}{S_{B H}^{\prime \prime}(E)}=-8 \pi E^{2} .
\end{aligned}
$$

Recently, black hole thermodynamics has been actively investigated in the non-extensive framework 33, 34, 35, 36, 37, 38, 39, 40, 41, 42], and the Rényi entropy formula has also been considered in this context [43, 44]. In this paper, our novel approach is to regard the Hawking-Bekenstein entropy as a Tsallis entropy, and use its additive formal logarithm, the Rényi entropy (7), for the canonical analysis instead. The essential difference is, that while the Tsallis entropy is not additive for factorizing probabilities, the Rényi entropy is, and thus, the zeroth law of thermodynamics apply. Via equations (7) and (17), one obtains the following result:

$$
\begin{aligned}
S_{R} & =\frac{1}{a} \ln \left(1+4 \pi a E^{2}\right) \\
\frac{1}{T_{R}} & =S_{R}^{\prime}(E)=\frac{8 \pi E}{1+4 \pi a E^{2}} \\
C_{R} & =\frac{-S_{R}^{\prime 2}(E)}{S_{R}^{\prime \prime}(E)}=\frac{8 \pi E^{2}}{4 \pi a E^{2}-1} .
\end{aligned}
$$

\section{Temperature and horizon radius}

By analyzing the thermodynamics given by the equations in (18), one realizes that it is surprisingly similar to the one of a black hole in AdS space 27. Consequently we now obtain simultaneously the Rényi-entropy based thermodynamics of the simple Schwarzschild black hole (discussed above) and the original Bekenstein-Hawking entropy based thermodynamics of a black hole in anti-de Sitter space. From now on we use the notation $E=M(r)$ with $r$ being the horizon radius.

In the first case we have the metric factor $f(r)=$ $1-2 M / r$ from which $M(r)=r / 2$ on the horizon. The considered entropy is the Rényi one, $S=\frac{1}{a} \ln \left(1+a \pi r^{2}\right)$. The temperature becomes

$$
T(r)=\frac{M^{\prime}(r)}{S^{\prime}(r)}=\frac{1}{4 \pi r}+\frac{a}{4} r .
$$

In the second case the metric factor is modified to $f(r)=$ $1-2 M / r+\lambda r^{2} / 2$ and

$$
M(r)=\frac{r}{2}\left(1+\lambda \frac{r^{2}}{2}\right) .
$$

Its radial derivative becomes

$$
M^{\prime}(r)=\frac{1}{2}+\lambda \frac{3 r^{2}}{4}
$$

giving rise to the temperature

$$
T(r)=\frac{M^{\prime}(r)}{S^{\prime}(r)}=\frac{1}{4 \pi r}+\frac{3 \lambda}{8 \pi} r .
$$

This intriguing coincidence motivates to consider a Rényi entropy with $a=3 \lambda / 2 \pi$ simulating the anti-de Sitter Boltzmannian thermodynamics for positive parameter values. In both cases there is a minimal temperature. The radius where this occurs satisfies $T^{\prime}\left(r_{0}\right)=0$, in the Rényi case $q=1+a=1+1 / \pi r_{0}^{2}$. At this radius the heat capacity has a pole:

$$
C(r)=\frac{d M}{d T}=\frac{M^{\prime}(r)}{T^{\prime}(r)}=\frac{2 \pi r_{0}^{2} r^{2}}{r^{2}-r_{0}^{2}} .
$$

The $q$-parameter for an ideal gas on the other hand is given as $q=1+1 / C_{0}$. This means that the characteristic ideal-gas-equivalent heat capacity, coded in this parameter, belongs to a positive $C_{0}=\pi r_{0}^{2}=S_{\mathrm{BH}}\left(r_{0}\right)$, actually not occurring in $C(r)$, since it stays over the asymptotic value $2 \pi r_{0}^{2}$ when positive.

\section{Black hole evaporation}

Classical Schwarzschild black hole horizons are bad heat containers due to their negative heat capacity. In the Rényi thermodynamics, or equivalently with the AdS metric, the heat capacity, $C=d M / d T=M^{\prime}(r) / T^{\prime}(r)$ is negative only for $r<r_{0}$, since in both cases $M(r)$ is monotonic rising, while $T(r)$ is falling for $r<r_{0}$ black hole horizons. At $r=r_{0}$ the heat capacity diverges, a pole occurs. Above $r_{0}$ the $T(r)$ curve is rising again featuring a positive heatcapacity system.

In the simplest evaporation scenario, the black-body radiation, belonging to the temperature $T(r)$, consumes the energy, $M(r)$, of the black hole. Using the StefanBoltzmann formula for the energy flux, one can model the energy loss as

$$
\dot{M}=M^{\prime}(r) \dot{r}=-4 \pi r^{2} \sigma T^{4}(r) .
$$

Since we have obtained the same functional form $T(r)$ for the Rényi-Schwarzschild and for the Boltzmann-AdS case, only the $M^{\prime}(r)$ factor distinguishes these cases. Due to the positivity of all factors, it is obvious that the lowest decay rate occurs roughly at $r=r_{0}$, so adiabatically radiating simple black holes spend the longest part of their life time near to this radius. The finite decay time, during which an initial radius, $R$, shrinks to zero, can be analytically obtained in both cases. It is given by

$$
t_{\text {life }}(R)=\frac{\left(4 \pi r_{0}\right)^{3}}{2 \sigma} \int_{0}^{R / r_{0}} \frac{x^{2}}{\left(1+x^{2}\right)^{n}} d x
$$




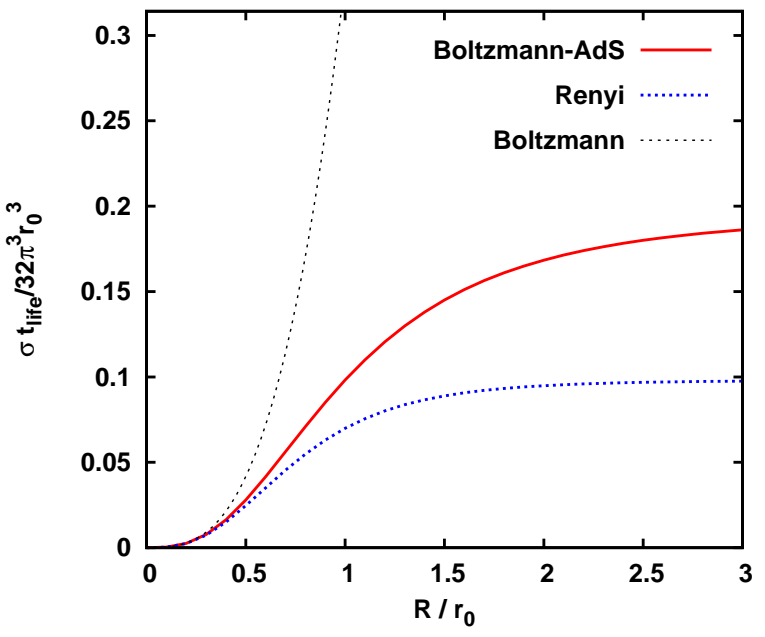

Figure 1: The scaled lifetime of spherical static black holes against black-body radiation in the Rényi-Schwarzschild and in the Boltzmann-AdS scenarios as functions of the initial radius scaled by the radius where the temperature is minimal, $R / r_{0}$.

with $n=3$ for the AdS and $n=4$ for the Rényi scenario. The analytic results of the integrations are

$$
t_{\mathrm{life}}^{\mathrm{AdS}}(R)=\frac{4 \pi^{3} r_{0}^{3}}{\sigma}\left[\arctan (x)+\frac{x\left(x^{2}-1\right)}{\left(x^{2}+1\right)^{2}}\right]
$$

for $n=3$ (AdS), and

$$
t_{\text {life }}^{\text {Renyi }}(R)=\frac{2 \pi^{3} r_{0}^{3}}{\sigma}\left[\arctan (x)+\frac{x\left(x^{4}+\frac{8}{3} x^{2}-1\right)}{\left(x^{2}+1\right)^{3}}\right]
$$

for $n=4$ (Rényi). Here we used the scaling notation $x=R / r_{0}$. For small initial radii $R \ll r_{0}$, both evolutions are well approximated by the classical Schwarzschild result

$$
t_{\text {life }}^{\text {class }}(R)=\frac{32 \pi^{3} R^{3}}{3 \sigma}
$$

However, very large black holes do not live forever in this approach: they also have a finite decay time. The maximal lifetime differs by a factor of two in these cases:

$$
t_{\text {life }}^{\text {Renyi }}(\infty)=\frac{\pi^{4} r_{0}^{3}}{\sigma}
$$

for the Rényi thermodynamics, while

$$
t_{\mathrm{life}}^{\mathrm{AdS}}(\infty)=\frac{2 \pi^{4} r_{0}^{3}}{\sigma}
$$

in the conventional thermodynamics of an AdS black hole. Figure 1 plots the entire evolution of the horizon radii.

\section{A semi-classical estimate for the $q$-parameter}

In particle physics, one conjectures small black holes, so $q$ may deviate from unity appreciably. For astronomical black holes it is probably practically unobservable (the maximal lifetime depending on the effective heat-capacity, $C\left(r_{1}\right)$, to which $q$ has been fitted to). In the followings we deal with an estimate for the horizon radius at the minimal temperature, $r_{0}$, for near-quantum black holes.

If the energy of such a state, $E_{0}=M\left(r_{0}\right)$, lied below or around the quantum mechanical ground state energy, then we have reached the edge of the classical theory discussed so far. Of course, in the absence of a functioning quantum theory of gravity, one can only have an estimate for this limit from semi-classical considerations. The simplest of which is regarding the energy of a string of the length of the diameter, $2 r_{0}$, having a wave number and frequency $\omega=k=\pi / 2 r_{0}$ (the sinus wave with no intermediate nodes and massless excitations). The corresponding ground state quantum oscillator energy is required to be greater than the classical black hole energy at the turning minimum point of the temperature:

$$
\frac{\omega}{2}=\frac{\pi}{4 r_{0}} \geq E_{0}=\frac{r_{0}}{2} .
$$

This translates to the condition $r_{0}^{2} \leq \pi / 2$. Utilizing now the relation $a=1 /\left(\pi r_{0}^{2}\right)$ one concludes that

$$
q=1+a \geq 1+\frac{2}{\pi^{2}} .
$$

It is amazing to realize that this estimate, $q \approx 1.2026$, is how well approximated by cosmic ray observations $(q=$ 11/9) [45, 46] and by the quark coalescence fit to RHIC hadron transverse momentum spectra $(q \approx 1.2)[19$.

\section{Summary}

By interpreting the Bekenstein-Hawking entropy as a non-extensive Tsallis entropy of Schwarzschild black hole horizons, and by considering their equation of state based on the Rényi entropy, one obtains a temperature minimum at a given horizon radius $r_{0}$. This feature (and the whole $T(r)$ curve) is of the same form as the result from a black hole in anti-de Sitter space by using the Boltzmann-Gibbs entropy. Requiring that the energy at this point, $E_{0}=$ $M\left(r_{0}\right)$, is above a semi-classical ground state of a string stretched over the diameter $2 r_{0}$ of the horizon, amazingly a Bekenstein bound for the $q$-parameter of Rényi's and Tsallis' entropy formulas arises. Findings in relativistic heavy ion collisions and in cosmic ray observations are characterized by a $q$-value surprisingly close to this bound.

Recently, black holes in AdS spacetimes seem to be relevant through the Maldacena conjecture [47], and it might be an interesting question whether our findings may be better understood or reinterpreted from the holographic gauge/gravity correspondence point of view [48, 49]. 
Although it is well known that micro black holes can not form in high energy particle and heavy ion collisions in 3-dimensions, nevertheless, due to the extreme deceleration by the stopping, a Rindler horizon may occur for the newly produced hadrons. This can be, in general, the origin of thermal looking spectra [50] as also suggested using different argumentation by Kharzeev and Satz earlier [51, 52]. This mechanism can well be the reason behind the leading non-extensive effect in the power-law spectra, consistent with a statistical, constituent quark matter hadronization picture.

In addition, it is also important to emphasize that for producing features analogue to the Hawking radiation in the spectrum, no black hole formation is needed in the classical sense. The formation of an event horizon suffices. As an example, it has been recently reported about ultrashort laser pulse experiments, that among other known radiations, a Hawking radiation of photons has also been produced [53].

Finally we would like to emphasize that if experimental findings would vastly violate the bound derived in eq. (32) on the quark level, - mesonic spectra showing half, while baryonic spectra one third of the $a=q-1$ quark level value - then it may be a strong argument against speculations on the presence of event horizon related phenomena in high energy particle collisions.

\section{Acknowledgement}

T.S.B. gratefully acknowledges discussions with Profs. C. Tsallis, B. Müller and A. Jakovác. V.G.Cz. has benefited from a discussion with Sameer Murthy. This work has been supported by Hungarian National Research Fund OTKA grant K104260, by a bilateral Hungarian-SouthAfrican project NIH TET_10-1_2011-0061 and ZA-15/2009, by the TÁMOP 4.2.1./B-09/1/KONV-2010-0007 project, co-financed by the European Union and European Social Fund, and was also partly supported by the Helmholtz International Center for FAIR within the framework of the LOEWE program (LandesOffensive zur Entwicklung Wissenschaftlich-Ökonomischer Exzellenz) launched by the State of Hesse. The research leading to these results has also received funding from the European Union Seventh Framework Programme (FP7/2007-2013) under grant agreement $n^{\circ}$ PCOFUND-GA-2009-246542 and from the Foundation for Science and Technology of Portugal.

\section{References}

[1] C. Tsallis, Introduction to Non-Extensive Statistical Mechanics: Approaching a Complex World, Springer, 2009.

[2] T. S. Biró, G. G. Barnaföldi and P. Ván, arXiv:1208.2533 (2012).

[3] T. S. Biró, Physica A 392 (2013) 3132.

[4] C. Tsallis, J. Stat. Phys. 52 (1988) 479

[5] C. Tsallis, Physica A 221 (1995) 277.

[6] C. Tsallis, Braz. J. Phys. 29 (1999) 1.

[7] C. Tsallis, Eur. Phys. J. A 40 (2009) 257.
[8] P. K. Kovtun, D. T. Son and A. O. Starinets, Phys. Rev. Lett. 94 (2005) 111601.

[9] D. Mateos, R. C. Myers, and R. M. Thomson, Phys. Rev. Lett. 97 (2006) 091601.

[10] D. Mateos, R. C. Myers, and R. M. Thomson, JHEP 05 (2007) 067.

[11] J. Noronha, M. Gyulassy and G. Torrieri, arXiv:0906.4099 (2009).

[12] J. M. Heuser et al, Acta Phys. Hung. Ser. A, Heavy Ion Phys. 15 (2002) 291

[13] K. Adcox et al, Phys. Rev. C 69 (2004) 024904.

[14] S. S. Adler et al, Phys. Rev. C 69 (2004) 034909; ibid. 69 (2004) 034910 .

[15] K. Reygers et al, Nucl. Phys. A 734 (2004) 74.

[16] C. Adler et al, Phys. Rev. Lett. 87 (2001) 112303.

[17] J. Adams et al, Phys. Rev. Lett. 91 (2003) 172302.

[18] A. A. P. Suaide et al, Braz. J. Phys. 34 (2004) 300.

[19] T. S. Biró and K. Ürmössy, J. Phys. G 36 (2009) 064044.

[20] T.S. Biró, G. Purcsel and K. Ürmössy, Eur. Phys. J. A 40 (2009) 325.

[21] A. Rényi, Acta Math. Acad. Sci. Hung. 10 (1959) 193.

[22] A. Renyi, Probability Theory, North Holland, Amsterdam, 1970.

[23] C. E. Shannon, Bell Syst. Tech. J. 27 (1948) 379; ibid. 27 (1948) 623.

[24] E. T. Jaynes, Phys. Rev. 160 (1957) 171; ibid. 168 (1957) 620.

[25] Z. Daróczy and J. Aczél, On Measures of Information and their Characterization, Academic Press, New York, 1975.

[26] J. H. Havrda and F. Charvat, Kybernetika 3 (1967) 30.

[27] S. W. Hawking and D. Page, Comm. Math. Phys. 87 (1983) 577.

[28] J. D. Bekenstein, Phys. Rev. D 7 (1973) 2333.

[29] J. M. Bardeen, B. Carter and S. W. Hawking, Comm. Math. Phys. 31 (1973) 161.

[30] S. W. Hawking, Comm. Math. Phys. 43 (1975) 199.

[31] J. D. Bekenstein, Phys. Rev. D 23 (1981) 287.

[32] W. G. Unruh, Phys. Rev. D 14 (1976) 870.

[33] P. T. Landsberg and D. Tranah, Phys. Lett. A 78 (1980) 219.

[34] P. T. Landsberg and N. T. Bishop, Gen. Rel. Grav. 19 (1987) 1083.

[35] P. T. Landsberg, J. Stat. Phys. 35 (1984) 159.

[36] P. T. Landsberg, Black Hole Physics, NATO Science Series, 364 (1992) 99.

[37] D. Pavón and J.M. Rubí, Gen. Rel. Grav. 18 (1986) 1245.

[38] J. Maddox, Nature, 365 (1993) 103.

[39] G. Gour, Class. Quant. Grav. 20 (2003) 3403.

[40] J. Oppenheim, Phys. Rev. E 68 (2003) 016108.

[41] A. Pesci, Class. Quant. Grav. 24 (2007) 2283.

[42] R.F. Aranha, H.P. de Oliveira, I. Damio Soares, E.V. Tonini, Int. J. Mod. Phys. D 17 (2008) 2049.

[43] A. Bialas and W. Czyz, Europhys. Lett. 83 (2008) 60009.

[44] A. Belin, A. Maloney and S. Matsuura, arXiv:1306.2640 (2013).

[45] C. Beck, Physica A 331 (2004) 173.

[46] C. Beck, Eur. Phys. J. A 40 (2009) 267.

[47] J. M. Malcadena, Adv. Theor. Math. Phys. 2 (1998) 231.

[48] E. Witten, Adv. Theor. Math. Phys. 2 (1998) 253.

[49] S. S. Gubser, I. R. Klebanov and A. M. Polyakov, Phys. Lett. B 428 (1998) 105.

[50] T. S. Biró, Z. Schram and M. Gyulassy, Phys. Lett. B 708 (2012) 276.

[51] D. Kharzeev and K. Tuchin, Nucl. Phys. A 753 (2005) 316.

[52] P. Castorina, D. Kharzeev and H. Satz, Eur. Phys. J. C 52 (2007) 187.

[53] F. Belgiorno, S. L. Cacciatori, M. Clerici, V. Gorini, G. Ortenzi, L. Rizzi, E. Rubino, V. G. Sala and D. Faccio, Phys. Rev. Lett. 105, (2010) 203901. 